\title{
Light-Activated Electrochemistry for the Two-Dimensional Interrogation of Electroactive Regions on a Monolithic Surface with Dramatically Improved Spatial Resolution
}

\author{
Ying Yang, ${ }^{\dagger}$ Simone Ciampi, ${ }^{\ddagger}$ Ying Zhu, ${ }^{\dagger}$ and J. Justin Gooding* ${ }^{*} \dagger$ \\ ${ }^{\dagger}$ School of Chemistry, The University of New South Wales, Sydney, NSW 2052, Australia \\ ${ }^{\ddagger}$ Intelligent Polymer Research Institute, University of Wollongong, Wollongong, NSW 2522, Australia
}

Supporting Information

ABSTRACT: The concept of light-activated electrochemistry (LAE) was recently presented where faradaic electrochemistry could be spatially resolved on a monolithic silicon electrode by illuminating the specific region with light. A major implication from the previous study using illumination from the nonsolution side, or backside, is that the spatial resolution is limited by the finite thickness of silicon wafer. To overcome this restriction, and enable the further application of LAE, in combination with optical imaging for example, herein the spatial resolution of LAE using topside illumination (illumination from the solution side) is explored. The applied potential and irradiated light intensity are found to have significant effects on the spatial resolution. A spatial resolution of $\sim 30 \mu \mathrm{m}$ was achieved with optimal parameters, which is a 20 times improvement compared with the previously reported backside illumination design, demonstrating the potential application of the strategy including microarray patterning of silicon or for single cell analysis.

\section{INTRODUCTION}

The concept of light-activated electrochemistry (LAE) was recently reported ${ }^{1}$ where on a poorly doped monolithic silicon surface faradaic electrochemistry could be performed only at the site of illumination. In this way, electrode arrays with only a single peripheral lead can connect multiple discrete conductive elements to an external circuit. The significance of this concept is that because the space occupied by connecting leads and bonding pads in conventional electrode arrays ${ }^{2,3}$ are no longer required, much higher density electrode arrays can in principle be fabricated. Furthermore, because no leads are required, the organization of the electrode array, that is, the position each electrode occupies in the array, no longer needs to be predetermined.

In previous work, the concept of LAE has been utilized for the mask-free electrochemical writing of polypyrrole arrays on a silicon surface and by operating a four-element/single-wire electrochemical sensor for reading DNA hybridization. ${ }^{1}$ In these initial examples, and mainly to simplify the illumination optics setup, a light pointer was focused and moved across the "dry" backside of the silicon electrode. This experimental simplification carries the intrinsic drawback of the achievable 2D resolution being limited by the isotropic diffusion ${ }^{4,5}$ of carriers in the electroneutral bulk of the electrode. A major implication is then the inverse relationship between spatial resolution and the finite thickness of a silicon electrode. For example, for a 200 $\mu \mathrm{m}$ thick wafer, which is still robust enough to allow for practical handling, a spatial resolution of only $\sim 300 \mu \mathrm{m}$ is obtained. ${ }^{1}$ An analogous experimental limitation was encoun- tered in previous research on light-addressable potentiometric sensors. ${ }^{6,7}$ Efforts to improve the spatial resolution may include the thinning of the substrate $e^{8,9}$ or applying illumination to generate charge carriers in the semiconductor space-charge only. ${ }^{10}$

Herein we explore topside illumination of the silicon electrode as it is expected to improve the spatial resolution of LAE, but without the need of any mechanical or chemical processing of the substrate. Furthermore, the topside illumination will make it possible to combine LAE with optical microscopy, thereby obtaining the optical and electrochemical images simultaneously. The experimental system employed to test the achievable spatial resolution is a poorly doped silicon photocathode that is first passivated against anodic decomposition by an alkyne-terminated organic monolayer. ${ }^{11,12}$ The monolayer is however thin enough to allow appreciable electron tunneling across it. To achieve LAE, there must also be sufficient electronic coupling when the redox active species are covalently bound to the alkyne layer. In our previous work, ${ }^{16}$ the LAE has been studied on an anthraquinoneterminated p-type silicon surface. Herein, using conventional lithographic procedures of the entire monolithic silicon electrode, only a thin strip of known width is chemically functionalized with an anthraquinone species. Similar to the way ferrocene modified silicon could assist the oxidation of

Received: March 3, 2016

Revised: $\quad$ May 5, 2016

Published: May 31, 2016 
ferrocyanide in solution, ${ }^{1}$ the attached anthraquinone units are capable of mediating a electrochemical reaction after applying a negative potential, i.e., oxygen reduction, ${ }^{14-13}$ while the alkyneterminated silicon is unable to perform the oxygen reduction. Thus, a rapid increase in the magnitude of reduction current is observed when the light source moves from the alkyne region to the anthraquinone region. The width of the anthraquinone pattern can then be measured by recording the current signal as a function of the known displacement of laser pointer when moving across the anthraquinone strip. In the present work, an improvement in spatial resolution refers to a better agreement between the experimental full width at half-maximum (FWHM) in the current/pointer-displacement curves and the actual known size of the anthraquinone pattern. The smallest feature resolved for silicon of $500 \mu \mathrm{m}$ thick used herein is 30 $\mu \mathrm{m}$ using topside illumination with optimal parameters, which is a 20 times improvement compared with the backside illumination setup.

\section{EXPERIMENTAL METHODS}

Materials. Hydrogen peroxide (30 wt \% in water, SigmaAldrich), hydrofluoric acid (48 wt \% in water, Riedel-Haën), and sulfuric acid (J.T. Baker) were of semiconductor grade. 1,8Nonadiyne (Aldrich, 98\%) was redistilled under reduced pressure $\left(65.0-69.9^{\circ} \mathrm{C}, 25-30\right.$ Torr) and stored under a dry argon atmosphere prior to use. 2-(Azidomethyl)anthracene9,10-dione was synthesized from commercially available 2(bromomethyl)anthracene-9,10-dione (Sigma-Aldrich, $\geq 97 \%$ ) following our previous work. ${ }^{16}$ The anhydrous solvents for surface cleaning were redistilled prior to use. Other chemicals used in preparation of buffer solutions, potassium chloride, sodium hydroxide, boric acid, phosphoric acid, and acetic acid are all of analytical grade and used as received. The p-type (boron-doped) silicon(100) wafers were of $500 \pm 25 \mu \mathrm{m}$ thickness and 10-20 $\Omega \mathrm{cm}$ resistivity and purchased from Siltronix, S.A.S. (Archamps, France).

Acetylene-Functionalized Silicon Surfaces. The acetylenylated silicon were prepared by covalent attachment of 1,8 nonadiyne followed previously reported procedure. ${ }^{11}$ The silicon samples approximately $20 \times 10 \mathrm{~mm}^{2}$ in size were rinsed in dichloromethane and ethanol, dried under a stream of argon, and then cleaned in a hot piranha solution of $1: 3(\mathrm{v} / \mathrm{v})$ $30 \%$ hydrogen peroxide to concentrated hydrofluoric acid at for $30 \mathrm{~min}$. Samples were then rinsed with water and etched with $2.5 \%$ aqueous hydrogen fluoride solution for $90 \mathrm{~s}$ to construct hydrogen-terminated surface. ${ }^{17}$ Silicon wafers were then transferred to 1,8-nonadiyne that was well degassed through multiple freezing and thawing cycles. The reaction was kept under an argon atmosphere in a Schlenk flask with set temperature of $165{ }^{\circ} \mathrm{C}$ for $3 \mathrm{~h}$. The obtained acetylenylated silicon wafers were then rinsed several times with ethanol and dichloromethane and stored under argon before further processing.

Fabrication of Microscale Anthraquinone on Acetylenylated Silicon Surface. As shown in Scheme 1a, the alkyneterminated silicon was first covered with positive photoresist AZ6632 (Microchemicals, Germany) by spin coating at 4000 $\mathrm{rpm}$ for $30 \mathrm{~s}$ and soft baking at $95{ }^{\circ} \mathrm{C}$ for $5 \mathrm{~min}$. The photoresist-coated substrate was irradiated with UV light for $7 \mathrm{~s}$ through a chrome-patterned photomask using a Quintel Q6000 mask aligner (Scheme 1b). Following UV exposure, the photoresist was immediately developed in AZMIF326 developer (Microchemicals, Germany) for $1 \mathrm{~min}$, rinsed with Milli-Q
Scheme 1. Anthraquinone Micropatterning on Acetylenylated Silicon Surface

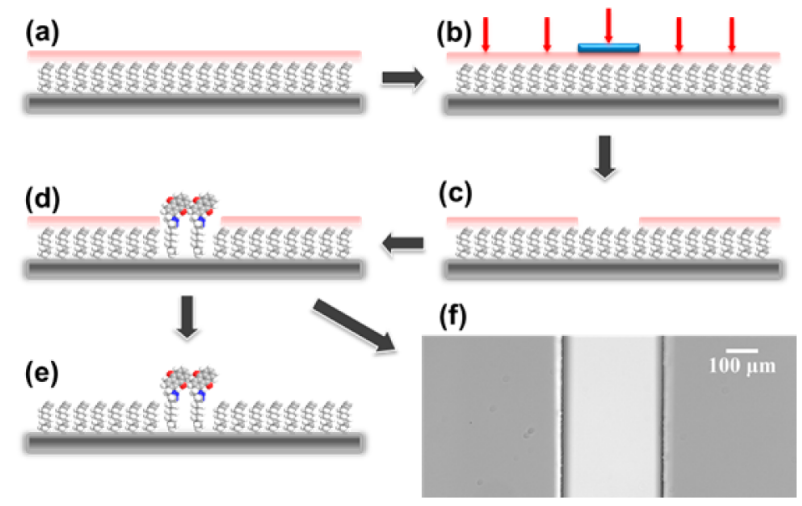

${ }^{a}$ Alkyne-terminated silicon is covered with photoresist. ${ }^{b}$ Photoresistcoated substrate is irradiated with UV light through a chromepatterned photomask. ${ }^{c}$ Photoresist is developed in AZMIF326 developer. ${ }^{d}$ Anthraquinone is attached on specified are via alkyneazide cycloaddition reaction. ${ }^{e}$ Photoresist is removed with dichloromethane. ${ }^{f}$ Direct observation of the anthraquinone pattern under microscope (before moving photoresist).

water for $30 \mathrm{~s}$, and dried under a stream of nitrogen gas (Scheme 1c). Anthraquinone drivative was covalently modified on acetylenylated silicon via $\mathrm{Cu}(\mathrm{I})$-catalyzed alkyne-azide cycloaddition to yield microscale anthraquinone patterning on silicon wafers. ${ }^{18,19}$ Specifically, alkyne-functionalized silicon surface were kept in $2 \mathrm{~mL}$ of dimethyl sulfoxide that contains $2 \mathrm{mM}$ 2-(azidomethyl)anthracene-9,10-dione, $10 \mathrm{mM}$ copper(I) bromide, and $20 \mathrm{mM}$ tetramethylethylenediamine at room temperature for $30 \mathrm{~min}$ (Scheme 1d). The prepared silicon with micropatterned anthraquinone were rinsed consecutively with copious amounts of water, ethanol, and dichloromethane to remove the photoresist and stored under dry argon before use (Scheme 1e). In this work, the patterned anthraquinone on silicon surfaces were prepared with different sizes $(15,30,50$, $80,200$, and $300 \mu \mathrm{m})$. Microscopy was performed on manual fluorescence microscope (Olympus BX53) equipped with a TH4-200 halogen light source for imaging. The microscopy image of silicon surface after anthraquinone "click" attachment and before removing photoresist is shown in Scheme 1f, in which an uncovered region with the width of $300 \mu \mathrm{m}$ was expected to be the modified with anthraquinone.

$\mathrm{X}$-ray photoelectron spectroscopy (XPS) measurements were performed with an ESCALAB 220i XL system to characterize the modification of silicon wafers. All reported energies were corrected based on the $\mathrm{C} 1 \mathrm{~s}$ signal at $285.0 \mathrm{eV}$. Thermo Avantage was used for peak component fitting.

Electrochemical Measurements. The electrochemical chamber sealed to the silicon with a gasket with a diameter of $5.8 \mathrm{~mm}$ was employed in all the electrochemical experiments; GaIn was applied on the back of silicon to make ohmic contacts. A three-electrode system was utilized, with a platinum mesh counter electrode and an $\mathrm{Ag} \mid \mathrm{AgCl} 1 \mathrm{M} \mathrm{KCl}$ reference electrode. Mott-Schottky plots were performed with a $\mathrm{CHI}$ 660D electrochemical workstation ( $\mathrm{CH}$ Instruments, USA). A $642 \mathrm{~nm}$ pigtailed laser diode (LP642-PF20, Thorlabs) mounted with a laser diode and temperature controller (ITC4001, Thorlabs) was employed. An X-Y motorized actuator (Z825B, Thorlabs) driven by the control cube (TDC001, Thorlabs) was utilized to drive the laser fiber across the pattern with 
controllable raster rate. A custom-made single mode fiber with mounted lens systems was connected with the laser diode to give a focused beam with the full width at half-maximum of $15.4 \mu \mathrm{m}$ (Gaussian fit). The output power was calibrated by a PM 20D digital power and energy meter with a S120C Si sensor (Thorlabs, USA) at the focusing distance of $21.5 \mathrm{~mm}$. The depth of electrolyte was maintained at $\sim 1 \mathrm{~cm}$, and the power loss during passing through the electrolyte is considered as negligible.

If not otherwise specified, experiments were performed in Britton Robinson (B\&R) buffer ${ }^{20}$ at $\mathrm{pH}$ 7.03. Chronoamperometry was performed in the air. Cyclic voltammetry were also carried out in three conditions to examine the $\mathrm{O}_{2}$ reduction catalytic property, which was deaerating for 20 min with argon of high-purity grade, deaerating for 20 min with $\mathrm{O}_{2}$ of highpurity grade, and leaving in the air condition. A Mott-Schottky plot was employed to determine $E_{\mathrm{fb}}$ from the relationship between the applied potential $E$ and the space charge capacitance $C_{\mathrm{sc}}$ using the Mott-Schottky equation ${ }^{21-23}$

$$
C_{\mathrm{sc}}{ }^{-2}=\frac{2}{\varepsilon \varepsilon_{0} q N A^{2}}\left(E-E_{\mathrm{fb}}-\frac{k T}{q}\right)
$$

where $\varepsilon$ is the dielectric constant for silicon, $\varepsilon_{0}$ the vacuum permittivity, $q$ the charge of the electron, $A$ the electrode surface area, $N$ the doping density of silicon, $E$ the applied potential, and $E_{\mathrm{fb}}$ the flat band potential, and at room temperature $k T / q$ is around $25 \mathrm{mV}$.

\section{RESULTS AND DISCUSSION}

XPS Characterization of Modified Silicon Surfaces. The alkyne-functionalized silicon surface has been well characterized in our past studies. ${ }^{11,12,24,25}$ The XPS spectra of alkyne-terminated silicon surface are shown in Figure S1 of the Supporting Information. Consistent with previous work, the bands of $\mathrm{Si}, \mathrm{C}$, and $\mathrm{O}$ were determined simultaneously on the full spectra. The peaks for $\mathrm{Si} 2 \mathrm{p}_{3 / 2}$ and $\mathrm{Si} 2 \mathrm{p}_{1 / 2}$ were shown in high resolution Si 2 p spectra at 99.5 and $100.1 \mathrm{eV}$, respectively. The absence of silicon oxidation peaks for the silicon modified with 1,8-nonadiyne was confirmed in the energy range 101$105 \mathrm{eV}^{26,27}$ Three $\mathrm{C}$ 1s peaks at 283.6 ( $\left.\mathrm{Si}-\mathrm{C}\right), 285.0$ (C-C), and $286.6 \mathrm{eV}(\mathrm{C}-\mathrm{O}$ or $\mathrm{C}-\mathrm{N})$ were assigned to the alkynederivatized silicon. ${ }^{24,25}$ The $\mathrm{Cu}(\mathrm{I})$-catalyzed cycloaddition reaction ("click" reaction) on alkyne-terminated silicon surface for grafting of azide-tagged molecules has been utilized extensively before. ${ }^{12,27,28}$ Here, anthraquinone attachment was realized with the "click" reaction either on the entire surface or at a specific micropatterned area. As expected, nitrogen content arises after "click" reaction from the XPS spectra in Figure 1. There is still no evidence of silicon oxidation on the $\mathrm{Si} 2 \mathrm{p}$ narrow scans acquired from the anthraquinone-modified surface. The $\mathrm{N}$ 1s spectra were fitted to two peaks with binding energies of 401.7 and $400.3 \mathrm{eV}$ with a $1: 2$ ratio of the integrated area, which were assigned to $\mathrm{N}-\mathrm{N}=\mathrm{N}$ and $\mathrm{C}-\mathrm{N}-$ $\mathrm{N}$, respectively. ${ }^{18,19,25}$ For the high-resolution C 1s spectra, the peaks at 283.8, 284.5, and $285.0 \mathrm{eV}$ were assigned to siliconbonded carbon and carbon-bonded carbon in aromatic and aliphatic configuration, respectively. Two more peaks at 288.0 and $286.5 \mathrm{eV}$ correspond to $\mathrm{C}=\mathrm{O}$ for carbonyl group and $\mathrm{C}-$ $\mathrm{N} / \mathrm{C}-\mathrm{O}$, which further confirmed the success of anthraquinone attachment on silicon. ${ }^{16,27}$

2D Electrochemical Reading Anthraquinone Patterning Strip. LAE was then utilized to read the width of
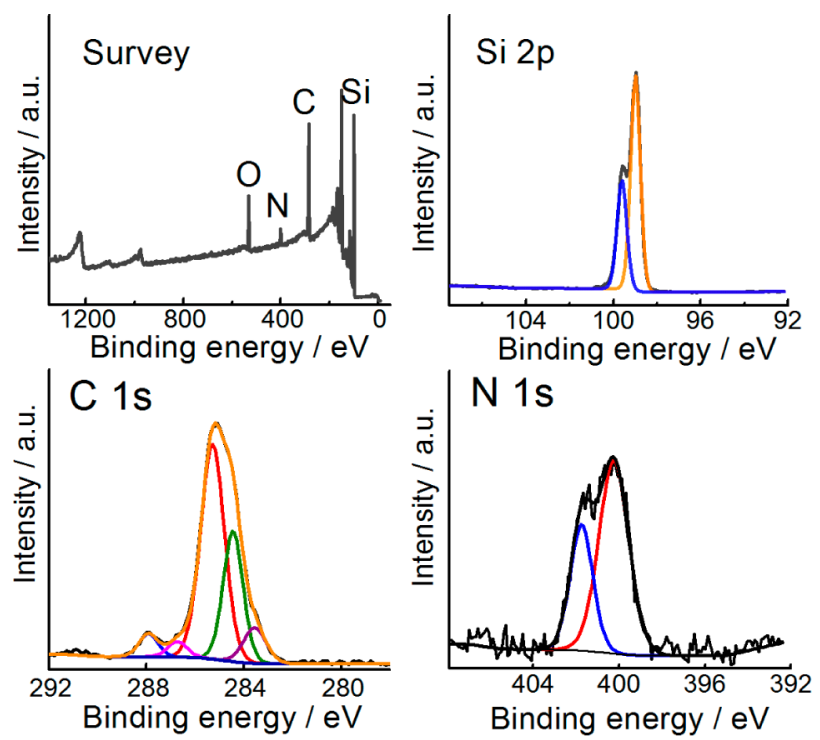

Figure 1. XPS survey spectra and high-resolution Si 2p, C 1s, and N 1s spectrographs of the anthraquinone-terminated silicon surface.

anthraquinone strip. As shown in Figure 2a, the silicon electrode with the anthraquinone pattern served as the working electrode, to which a negative potential was applied. The illumination optics was driven across the surface at a specific raster rate $\left(200 \mu \mathrm{m} \mathrm{s}^{-1}\right)$ along a direction intersecting the anthraquinone pattern. When the laser illuminates the silicon, electrons excited into the conduction band populate the interface and the illuminated site becomes transiently conductive (i.e., kinetic limitations of the depleted dark electrode are temporarily lifted). The current and laser displacement were collected simultaneously to build a current-distance $(I-d)$ trace (Figure 2b). When the anthraquinone-terminated area is under irradiation, anthraquinone will be reduced and serve as a redox mediator for the reduction of oxygen solution, and the electrocatalytic property was confirmed using cyclic voltammograms (Figure S2). ${ }^{14-13}$ Hence, the $I-d$ traces show a very low background current (approximately $0.1 \mathrm{nA}$ ) when the alkyne-terminated region is in the light path but with a steep rise in the faradaic current when the anthraquinone pattern begins to be illuminated. Note there was no evidence that the anthraquinone itself was photoactivated by the $640 \mathrm{~nm}$ laser light source. The geometrical characteristics, namely the width of the anthraquinone features, are then revealed by the $I-d$ data (Figure $2 b$ ), with the experimental FWHM being a perfect match to the known width $(300 \mu \mathrm{m})$ of the anthraquinone strip.

The effects of the dissolved oxygen concentrations in the electrolytes on the FWHM in $I-d$ curves were also compared, and the results are shown in Figure $2 \mathrm{~b}$. It was observed that the presence of increased dissolved oxygen resulted in an increased current at the AQ-modified silicon/electrolyte interface, but the FWHM, and hence the inferred spatial resolution, remained unchanged.

Subsequently, the FWHM with the same setup, but utilizing backside illumination, was investigated at the same $500 \mu \mathrm{m}$ thick silicon substrate. Figure $2 \mathrm{c}$ presents how the experimental FWHM with backside illumination is $680 \mu \mathrm{m}$. This spatial resolution is much greater than the actual value of patterning width $(300 \mu \mathrm{m})$ or the FWHM obtained from topside illumination $(300 \mu \mathrm{m})$. Hence, the preliminary result reveals 
(a)

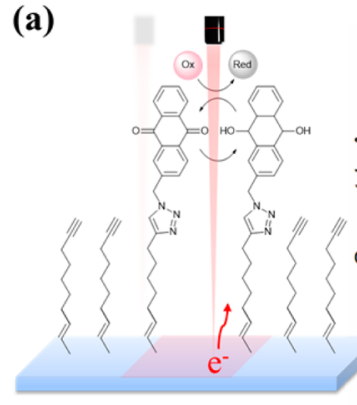

(b)

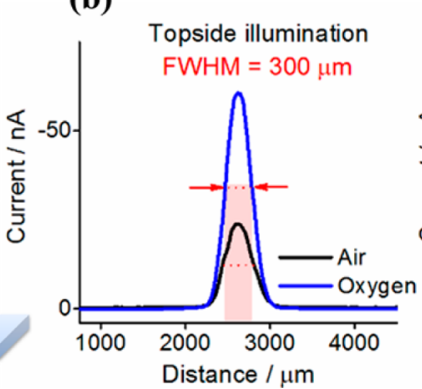

(c)

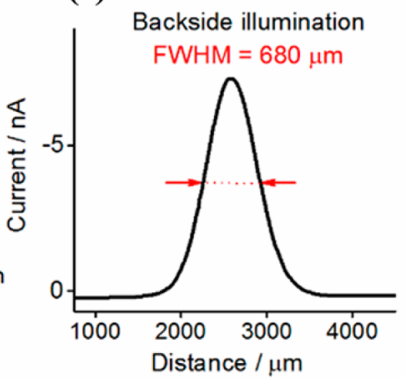

Figure 2. 2D electrochemical reading through a single peripheral lead. (a) Depiction of the LAE set up with an anthraquinone strip of known width. Current versus distance trace for an anthraquinone strip $300 \mu \mathrm{m}$ in width in $\mathrm{B} \& \mathrm{R}$ buffer $(\mathrm{pH} 7.03$ ) (b) under air or deaerating with oxygen with topside illumination and (c) under air with backside illumination. The applied potential was $-0.4 \mathrm{~V}$ vs reference, the laser raster rate was $200 \mu \mathrm{m} / \mathrm{s}$, and the light intensity was set to $1 \mathrm{~W} \mathrm{~cm}^{-2}$.

a clear advantage to using topside illumination to localize the faradaic reaction on a monolithic silicon electrode.

The lower limit of the spatial resolution was investigated by progressively reducing the width of the anthraquinone strip and measuring the FWHM from the corresponding $I-d$ curves. As shown in Figure 3, experimental FWHM's were close to the
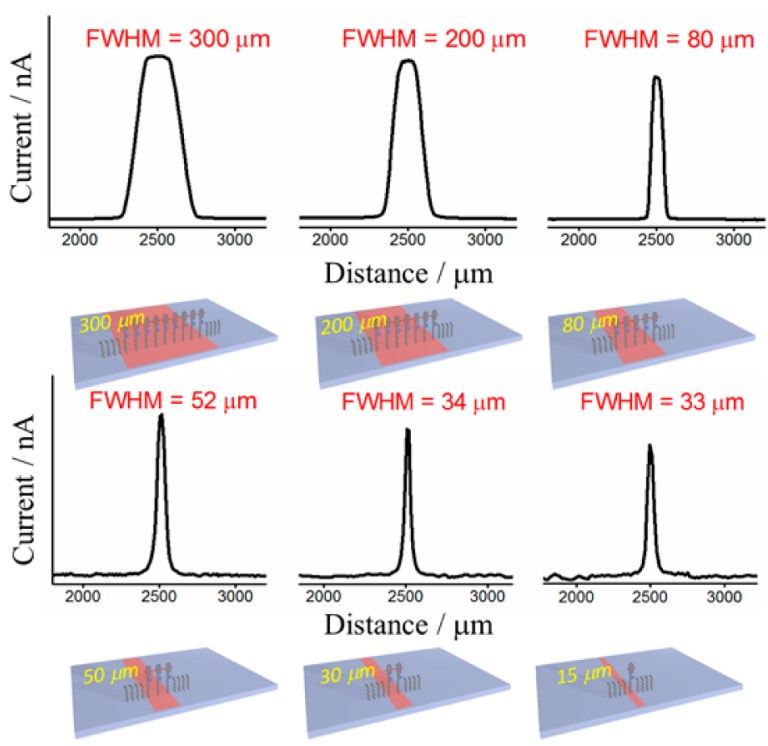

Figure 3. Current vs light pointer position for LAE photocathodes having different anthraquinone patterning sizes (300 to $15 \mu \mathrm{m}$ ). Raster rate was $200 \mu \mathrm{m} / \mathrm{s}$, and light intensity was $1 \mathrm{~W} \mathrm{~cm}^{-2}$. Note the magnitudes of cathodic current values were obviously reduced with size decrease of anthraquinone patterning area, and hence $Y$-axis was not drawn to the same scale for easy FWHM comparison.

actual pattern size down to $30 \mu \mathrm{m}$ and only started to diverge when the feature was $15 \mu \mathrm{m}$. These FWHM values have been extensively reproduced, thus exhibiting excellent analytical robustness of the LAE system. When the data for the smallest anthraquinone patterns are considered, the topside LAE device shows a 20 times improvement from the backside set up of silicon photocathodes of the same substrate thickness. This advantage was further demonstrated by light-assisted electrochemical "writing" conducting polymeric features on a homogeneous alkyne terminated n-type silicon electrode (Figure S3). It was achieved by electrodepositing polyaniline from aniline monomers on silicon electrode. Compared with previous reported spatial resolution of $\sim 400 \mu \mathrm{m},{ }^{1}$ a polymer patterning with width of $\sim 20 \mu \mathrm{m}$ was obtained, thereby confirming the improved resolution of topside LAE.

According to previous reports, the width of the space charge layer for a poorly doped p-type silicon electrode is typically less than $1-2 \mu \mathrm{m} .{ }^{29-31}$ For red laser illumination (with wavelength of $640 \mathrm{~nm}$ ), the charge carriers are only effectively generated to within $1 \mu \mathrm{m}$ of the surface. ${ }^{32}$ Thus, with topside illumination, appreciable charge carriers are being generated in the space charge region where they will drift to the interface under the electric field. ${ }^{33}$ Hence, depending on the experimental conditions, the generated charge carriers can be extracted efficiently to the interface as soon as generated. In contrast, with backside illumination, the photogenerated charge carriers must diffuse through the bulk silicon to the depletion region $^{34-36}$ whereupon electron transfer can be achieved at the silicon/electrolyte interface. As this electron diffusion can also occur laterally, the region with photogenerated carriers broadens and hence spatial resolution is inferior and typically is similar to the thickness of the silicon. ${ }^{6,7}$

Effects of Light Intensity on Spatial Resolution. If, as we propose, the superior spatial resolution of the topside illumination is because the charge carriers are being generated where needed, then the spatial resolution should be influenced by the light intensity. This is what is shown in Figure 4 with

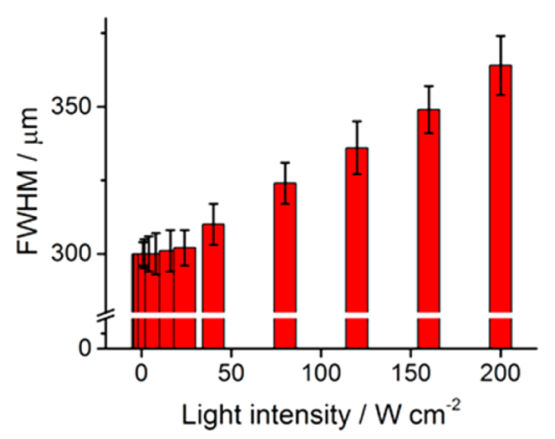

Figure 4. FWHM variation with increased light intensity at silicon electrode with anthraquinone strip width of $300 \mu \mathrm{m}$, when the applied potential is $-0.4 \mathrm{~V}$.

both an increase in FWHM for a $300 \mu \mathrm{m}$ anthraquinone stripe and an increase in faradaic current (Figure S4). The observed higher reduction current can be attributed to the increased minority carrier density with greater light intensity. Regarding the spatial resolution, Figure 4 shows that the FWHM remains at $\sim 300 \mu \mathrm{m}$ within the light intensity range of $1-20 \mathrm{~W} \mathrm{~cm}^{-2}$. 
However, the FWHM increases linearly with intensity higher than $20 \mathrm{~W} \mathrm{~cm}^{-2}$.

We propose a combination of two effects for the FWHM varying with light intensity. The first is that it has been shown previously that the photogenerated carrier lifetime is dependent on light intensity. ${ }^{37-39}$ That is, the electron diffusion length increases with light intensity and any such diffusion will cause a broadening of the FWHM. Second, the space charge layer width is known to decrease with increasing light intensity and the bands tend to flatten at higher light intensities. ${ }^{40,41}$ If the space charge becomes thin in comparison to the penetration depth of the light, then generation of charge carriers will occur in the bulk region of the semiconductor where they can again diffuse back to the interface and cause broadening of the FWHM. In order to restrict carrier diffusion and restrict carriers generation beyond the depletion region, and thus to optimize the spatial resolution of the LAE system, the experimental results presented in Figures 2 and 3 were conducted under light intensity of $1 \mathrm{~W} \mathrm{~cm}{ }^{-2}$.

Dependence of Spatial Resolution on Applied Potential. Finally, the influence of the applied potential on the spatial resolution was explored. As mentioned in the previous section, the light penetration depth is greater than the depletion layer width; the photocurrent is then mainly influenced by the potential drop across the deletion layer. ${ }^{30,42}$ Consequently, there is significant increase in the magnitude of the cathodic current with more negative potentials (see Figure S5 for $I-d$ curves). The FWHM observed under different potentials increased slightly at potentials less negative $-0.4 \mathrm{~V}$ and then significantly increased at more cathodic potential, thus giving inferior spatial resolution (Figure 5a). Given the above

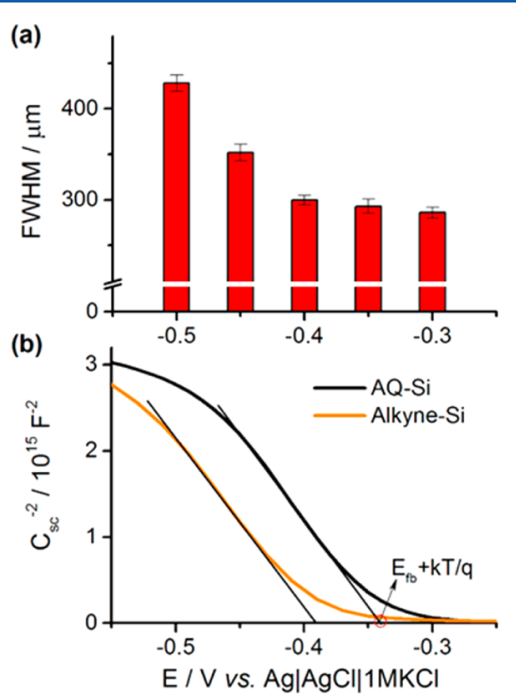

Figure 5. (a) Effects of applied potential on FWHM at silicon electrode with an anthraquinone strip (width of $300 \mu \mathrm{m}$ ). Light intensity was $1 \mathrm{~W} \mathrm{~cm} \mathrm{~cm}^{-2}$. (b) Mott-Schottky plots of anthraquinone and alkyne functionalized poorly doped p-type silicon electrodes in $\mathrm{B} \& \mathrm{R}$ buffer solution at $\mathrm{pH} 7.03$. Frequency was $10 \mathrm{kHz}$.

discussion, the improved spatial resolution using topside illumination was mainly attributed to the considerable photogeneration of charge carriers within the space charge region, in which the charge carriers were efficiently separated and bulk diffusion was restricted. Since the depletion width is dependent on the potential drop across this layer, ${ }^{43-45}$ thus any parameter that alters the band bending will vary the fractions of carriers generated in the depletion layer. However, the result in FWHM versus applied potential shows FWHM increases concomitant with more negative applied potential or with the increase of width of depletion region, which seems conflicts with the prediction.

The impact of potential thus introduces the other possible determinants in the spatial resolution achievable with LAE; the band bending status of the silicon electrode and changes to the electron drift velocity with electric field strength. To determine the bands position, direction, and magnitude of band bending, the flat band potentials $\left(E_{\mathrm{fb}}\right)$ were measured by Mott-Schottky plots $^{21-23,46}$ on the anthraquinone and alkyne-functionalized ptype silicon electrodes, and the results are shown in Figure $5 \mathrm{~b}$. The measured $E_{\mathrm{fb}}$ for the anthraquinone and alkyne modified ptype silicon electrode is -0.36 and $-0.41 \mathrm{~V}$, respectively. Through comparison of Figures $5 \mathrm{a}$ and $5 \mathrm{~b}$, it has been shown that when the applied potential is more positive than $E_{\mathrm{fb}}$, the electrode is under accumulation and applied potential makes little difference to the spatial resolution. Moreover, it seems that $-0.4 \mathrm{~V}$, where alkyne-terminated regions are in accumulation and the anthraquinone regions are in depletion, is a sweet spot to achieve the best spatial resolution. At such an applied potential, there will be sufficient potential for electron drift toward the interface under the anthraquinone regions, but the photogenerated carriers under the alkyne regions cannot drift to the interface. Furthermore, when the applied potential is more negative than the $E_{\mathrm{fb}}$ of both anthraquinone and alkynederivatized silicon, the silicon was always under depletion, and FWHM was found to be much larger than the actual value of patterning width. Since the drift velocity of carriers increases with the potential drop across the depletion region, ${ }^{33,47-49}$ the photogenerated electrons under alkyne drift toward the interface and diffuse to the nearby anthraquinone; hence, the observed result indicates the higher carrier velocity can further broaden FWHM on $I-d$ traces. For these above-mentioned reasons, an applied potential of $-0.4 \mathrm{~V}$ is used for all results above.

\section{CONCLUSIONS}

In summary, by taking the advantages of LAE on poorly doped p-type silicon photocathodes, a strategy for improving spatial resolution was developed using topside illumination. This strategy makes use of traveling light pointer to localize a faradaic reaction, hence allowing building a current 2D map with only one physical contact between the sample and a potentiostat. The understanding of light intensity/applied potential effect on spatial resolution offers significant importance for practical application of LAE. Using the optimal combination of these variables, the system achieves a spatial resolution of $\sim 30 \mu \mathrm{m}$, which is a 20 -fold improvement compared with backside illumination under the same conditions. This is extremely important, as topside illumination will make it possible for rapid integration of LAE with conventional optical microscopes. The versatility of the LAE probe will be further explored on silicon-based sensors for optically resolved single cell isolation, and the related work is in progress.

\section{ASSOCIATED CONTENT}

\section{Supporting Information}

The Supporting Information is available free of charge on the ACS Publications website at DOI: 10.1021/acs.jpcc.6b02289. 
XPS data for alkyne-passivated silicon surface, supplementary cyclic voltammograms and $I-d$ curves, and application of LAE for polymer deposition using topside illumination (PDF)

\section{AUTHOR INFORMATION}

\section{Corresponding Author}

*E-mail justin.gooding@unsw.edu.au; Ph +61-2-9385 5384 (J.J.G.).

\section{Notes}

The authors declare no competing financial interest.

\section{ACKNOWLEDGMENTS}

Dr. Peter Reece is thanked for useful suggestions in optical experimental. We thank Dr. Stephen Parker and Dr. Alexander $\mathrm{H}$ Soeriyadi for performing the XPS measurements. Yong $\mathrm{Lu}$ is thanked for three-dimensional figure drawing. This research was supported by the Australian Research Council's Discovery Projects Funding Scheme (DP150103065) and the Centre of Excellence for Convergent Bio-Nano Science and Technology (CE140100036).

\section{REFERENCES}

(1) Choudhury, M. H.; Ciampi, S.; Yang, Y.; Tavallaie, R.; Zhu, Y.; Zarei, L.; Goncales, V. R.; Gooding, J. J. Connecting Electrodes with Light: One Wire, Many Electrodes. Chem. Sci. 2015, 6, 6769-6776.

(2) Huang, X.-J.; O’Mahony, A. M.; Compton, R. G. Microelectrode Arrays for Electrochemistry: Approaches to Fabrication. Small 2009, 5, $776-788$.

(3) Lin, Z.; Ino, K.; Shiku, H.; Matsue, T. Electrochemical Topography of a Cell Monolayer with an Addressable Microelectrode Array. Chem. Commun. 2010, 46, 559-561.

(4) Robson, R. Diffusivity of Charge Carriers in Semiconductors in Strong Electric Fields. Phys. Rev. Lett. 1973, 31, 825.

(5) Uren, M.; Day, D.; Kirton, M. 1/F and Random Telegraph Noise in Silicon Metal-Oxide-Semiconductor Field-Effect Transistors. Appl. Phys. Lett. 1985, 47, 1195-1197.

(6) George, M.; Parak, W. J.; Gerhardt, I.; Moritz, W.; Kaesen, F.; Geiger, H.; Eisele, I.; Gaub, H. E. Investigation of the Spatial Resolution of the Light-Addressable Potentiometric Sensor. Sens. Actuators, A 2000, 86, 187-196.

(7) Sartore, M.; Adami, M.; Nicolini, C.; Bousse, L.; Mostarshed, S.; Hafeman, D. Minority Carrier Diffusion Length Effects on LightAddressable Potentiometric Sensor (Laps) Devices. Sens. Actuators, A 1992, 32, 431-436.

(8) Hafeman, D. G.; Parce, J. W.; McConnell, H. M. LightAddressable Potentiometric Sensor for Biochemical Systems. Science 1988, 240, 1182-1185.

(9) Owicki, J. C.; Bousse, L. J.; Hafeman, D. G.; Kirk, G. L.; Olson, J. D.; Wada, H. G.; Parce, J. W. The Light-Addressable Potentiometric Sensor: Principles and Biological Applications. Annu. Rev. Biophys. Biomol. Struct. 1994, 23, 87-114.

(10) Parak, W. J.; Hofmann, U. G.; Gaub, H. E.; Owicki, J. C. Lateral Resolution of Light-Addressable Potentiometric Sensors: An Experimental and Theoretical Investigation. Sens. Actuators, A 1997, 63, 4757.

(11) Ciampi, S.; Eggers, P. K.; Le Saux, G.; James, M.; Harper, J. B.; Gooding, J. J. Silicon (100) Electrodes Resistant to Oxidation in Aqueous Solutions: An Unexpected Benefit of Surface Acetylene Moieties. Langmuir 2009, 25, 2530-2539.

(12) Ciampi, S.; Harper, J. B.; Gooding, J. J. Wet Chemical Routes to the Assembly of Organic Monolayers on Silicon Surfaces Via the Formation of Si-C Bonds: Surface Preparation, Passivation and Functionalization. Chem. Soc. Rev. 2010, 39, 2158-2183.

(13) Yang, Y.; Ciampi, S.; Choudhury, M. H.; Gooding, J. J. Light Activated Electrochemistry: Light Intensity and $\mathrm{pH}$ Dependence on
Electrochemical Performance of Anthraquinone Derivatized Silicon. J. Phys. Chem. C 2016, 120, 2874-2882.

(14) Mirkhalaf, F.; Tammeveski, K.; Schiffrin, D. J. Substituent Effects on the Electrocatalytic Reduction of Oxygen on Quinonemodified Glassy Carbon Electrodes. Phys. Chem. Chem. Phys. 2004, 6, $1321-1327$.

(15) Vaik, K.; Mäeorg, U.; Maschion, F. C.; Maia, G.; Schiffrin, D. J.; Tammeveski, K. Electrocatalytic Oxygen Reduction on Glassy Carbon Grafted with Anthraquinone by Anodic Oxidation of a Carboxylate Substituent. Electrochim. Acta 2005, 50, 5126-5131.

(16) Kullapere, M.; Tammeveski, K. Oxygen Electroreduction on Anthraquinone-Modified Nickel Electrodes in Alkaline Solution. Electrochem. Commun. 2007, 9, 1196-1201.

(17) Pleskov, Y. V.; Zhang, G. Electrochemistry of Silicon and Its Oxide. Russ. J. Electrochem. 2002, 38, 454-455.

(18) Devadoss, A.; Chidsey, C. E. D. Azide-Modified Graphitic Surfaces for Covalent Attachment of Alkyne-Terminated Molecules by "Click" Chemistry. J. Am. Chem. Soc. 2007, 129, 5370-5371.

(19) Rohde, R. D.; Agnew, H. D.; Yeo, W.-S.; Bailey, R. C.; Heath, J. R. A Non-Oxidative Approach toward Chemically and Electrochemically Functionalizing Si(111). J. Am. Chem. Soc. 2006, 128, 95189525.

(20) Britton, H. T. S.; Robinson, R. A. Universal Buffer Solutions and the Dissociation Constant of Veronal. J. Chem. Soc. 1931, 0, 14561462.

(21) Cooper, G.; Turner, J. A.; Nozik, A. J. Mott-Schottky Plots and Flat-Band Potentials for Single-Crystal Rutile Electrodes. J. Electrochem. Soc. 1982, 129, 1973-1977.

(22) Rettie, A. J.; Lee, H. C.; Marshall, L. G.; Lin, J.-F.; Capan, C.; Lindemuth, J.; McCloy, J. S.; Zhou, J.; Bard, A. J.; Mullins, C. B. Combined Charge Carrier Transport and Photoelectrochemical Characterization of $\mathrm{BiVO}_{4}$ Single Crystals: Intrinsic Behavior of a Complex Metal Oxide. J. Am. Chem. Soc. 2013, 135, 11389-11396.

(23) Singh, P.; Singh, R.; Gale, R.; Rajeshwar, K.; DuBow, J. Surface Charge and Specific Ion Adsorption Effects in Photoelectrochemical Devices. J. Appl. Phys. 1980, 51, 6286-6291.

(24) Ciampi, S.; James, M.; Darwish, N.; Luais, E.; Guan, B.; Harper, J. B.; Gooding, J. J. Oxidative Acetylenic Coupling Reactions as a Surface Chemistry Tool. Phys. Chem. Chem. Phys. 2011, 13, 1562415632.

(25) Ciampi, S.; Boecking, T.; Kilian, K. A.; James, M.; Harper, J. B.; Gooding, J. J. Functionalization of Acetylene-Terminated Monolayers on $\mathrm{Si}(100)$ Surfaces: A Click Chemistry Approach. Langmuir 2007, 23, 9320-9329.

(26) Wallart, X.; Henry de Villeneuve, C.; Allongue, P. Truly Quantitative Xps Characterization of Organic Monolayers on Silicon: Study of Alkyl and Alkoxy Monolayers on $\mathrm{H}-\mathrm{Si}(111)$. J. Am. Chem. Soc. 2005, 127, 7871-7878.

(27) Ciampi, S.; James, M.; choudhury, M.; Darwish, N.; Gooding, J. J. The Detailed Characterization of Electrochemically Switchable Molecular Assemblies on Silicon Electrodes. Phys. Chem. Chem. Phys. 2013, 15, 9879-9890.

(28) Ciampi, S.; Bocking, T.; Kilian, K. A.; Harper, J. B.; Gooding, J. J. Click Chemistry in Mesoporous Materials: Functionalization of Porous Silicon Rugate Filters. Langmuir 2008, 24, 5888-5892.

(29) Krishnan, R. Fundamentals of Semiconductor Electrochemistry and Photoelectrochemistry; Wiley-VCH: Weinheim, 2007.

(30) Pleskov, Y. V.; Gurevich, Y. Y. Semiconductor Photoelectrochemistry; Consultants Bureau: New York, 1986.

(31) Chazalviel, J. N. Electrochemical Transfer Via Surface States: A New Formulation for the Semiconductor/Electrolyte Interface. J. Electrochem. Soc. 1982, 129, 963-969.

(32) Green, M. A.; Keevers, M. J. Optical Properties of Intrinsic Silicon at $300 \mathrm{~K}$. Prog. Photovoltaics 1995, 3, 189-192.

(33) Canali, C.; Majni, G.; Minder, R.; Ottaviani, G. Electron and Hole Drift Velocity Measurements in Silicon and Their Empirical Relation to Electric Field and Temperature. IEEE Trans. Electron Devices 1975, 22, 1045-1047. 
(34) Moritz, W.; Yoshinobu, T.; Finger, F.; Krause, S.; MartinFernandez, M.; Schöning, M. J. High Resolution Laps Using Amorphous Silicon as the Semiconductor Material. Sens. Actuators, $B$ 2004, 103, 436-441.

(35) Zimmermann, W. Measurement of Spatial Variations of the Carrier Lifetime in Silicon Power Devices. Phys. Status Solidi 1972, 12, 671-678.

(36) George, M.; Parak, W.; Gerhardt, I.; Moritz, W.; Kaesen, F.; Geiger, H.; Eisele, I.; Gaub, H. Investigation of the Spatial Resolution of the Light-Addressable Potentiometric Sensor. Sens. Actuators, A 2000, 86, 187-196.

(37) Zhang, W.; Lehmann, S.; Mergenthaler, K.; Wallentin, J.; Borgström, M. T.; Pistol, M.-E.; Yartsev, A. Carrier Recombination Dynamics in Sulfur-Doped InP Nanowires. Nano Lett. 2015, 15, $7238-7244$.

(38) Shimokawa, R; Hayashi, Y. Effect of Illumination Wavelength and Intensity on Minority-Carrier Diffusion Length of Efg Silicon Ribbon Solar Cells. IEEE Trans. Electron Devices 1983, 30, 1770-1775.

(39) Barnes, P. R. F.; Anderson, A. Y.; Koops, S. E.; Durrant, J. R.; O'Regan, B. C. Electron Injection Efficiency and Diffusion Length in Dye-Sensitized Solar Cells Derived from Incident Photon Conversion Efficiency Measurements. J. Phys. Chem. C 2009, 113, 1126-1136.

(40) Bocarsly, A. B.; Walton, E. G.; Bradley, M. G.; Wrighton, M. S. Two-Electron Oxidations at Illuminated N-Type Semiconducting Silicon Electrodes: Use of Chemically Derivatized Photoelectrodes. J. Electroanal. Chem. Interfacial Electrochem. 1979, 100, 283-306.

(41) Bolts, J. M.; Wrighton, M. S. Chemically Derivatized N-Type Semiconducting Gallium Arsenide Photoelectrodes. Thermodynamically Uphill Oxidation of Surface-Attached Ferrocene Centers. J. Am. Chem. Soc. 1979, 101, 6179-6184.

(42) Butler, M. Photoelectrolysis and Physical Properties of the Semiconducting Electrode $\mathrm{WO}_{2}$. J. Appl. Phys. 1977, 48, 1914-1920.

(43) Sze, S. M.; Ng, K. K. Physics of Semiconductor Devices; Wiley-

VCH: Weinheim, 2006

(44) Baccarani, G.; Ricco, B.; Spadini, G. Transport Properties of Polycrystalline Silicon Films. J. Appl. Phys. 1978, 49, 5565-5570.

(45) Takshi, A.; Dimopoulos, A.; Madden, J. D. Depletion Width Measurement in an Organic Schottky Contact Using a MetalSemiconductor Field-Effect Transistor. Appl. Phys. Lett. 2007, 91, 083513.

(46) Faber, E. J.; Sparreboom, W.; Groeneveld, W.; de Smet, L. C. P. M.; Bomer, J.; Olthuis, W.; Zuilhof, H.; Sudholter, E. J. R.; Bergveld, P.; van den Berg, A. Ph Sensitivity of Si-C Linked Organic Monolayers on Crystalline Silicon Surfaces. ChemPhysChem 2007, 8, 101-112.

(47) Chen, Y.-F.; Fuhrer, M. Electric-Field-Dependent ChargeCarrier Velocity in Semiconducting Carbon Nanotubes. Phys. Rev. Lett. 2005, 95, 236803.

(48) Canali, C.; Ottaviani, G.; Quaranta, A. A. Drift Velocity of Electrons and Holes and Associated Anisotropic Effects in Silicon. J. Phys. Chem. Solids 1971, 32, 1707-1720.

(49) Canali, C.; Jacoboni, C.; Nava, F.; Ottaviani, G.; AlberigiQuaranta, A. Electron Drift Velocity in Silicon. Phys. Rev. B 1975, 12, 2265. 